\title{
Waiting for Kids and Access to Quality Preventive Care: Her Fertility depends on it
}

\author{
Takiyah W Ndwanya* \\ Walden University, USA
}

Submission: April 27, 2017; Published: May 8, 2017

*Corresponding author: Takiyah W Ndwanya, PhD, MPH Walden University, Minneapolis, USA, Tel: +15713529787;

Email: Takiyah.White@gmail.com

\section{Background}

Women are deciding to have children later on in life. In the United States, the mean age of mothers having her first child increased from 24.9 years in the year 2000 to 26.3 years in 2014 [1]. With increasing age of first pregnancy, the composition and growth of the U.S. population impacts multiple birth pregnancies, and potential congenital disabilities [1]. Women aged 30-34 had the greatest gain of first births at 28\% from the years 2000-2014 from $16.5 \%$ to $21.1 \%$ [1]. One reason for this is due to women pursuing careers before having a family. While some may argue that this is not a concern or issue in the developing world, women deciding to have children at a later stage of life after starting careers and completing advanced education find out that lack of access to preventative services and past sexual activities may have influenced their ability to reproduce.

While not scientifically proven, the concept of 'leaning in' coined by Facebook Chief Operating Officer, Sheryl Sandberg in her book, "Lean In: Women, Work, and the Will to Lead," explores the idea of actively pursuing careers as women whether having a family or not. 'Leaning In' as a social trend has lent support for those who want to prioritize a job and make it to the top of her field or achieve any goal vigorously [2]. Sandberg makes the statement that "if we can succeed in adding more female voices at the highest levels, we will expand opportunities and extend fairer treatment to all" [2].

The Demographic Transition Theory, based in the social sciences, is a theory that societies progress from pre-modern times of high fertility and high mortality to a post-modern era of low fertility and low mortality [3]. The transition signifies a reduction in the death rate by controlling epidemic and contagious diseases; with modernization, children become more costly, and empowerment of women with advancing education to make reproductive decisions lead to smaller families [3]. A study based at the ESRC Centre for Population Change at the University of Southampton in Britain revealed that an influencing factor of women having their first child later in life is to finish full-time education and training at a later age [4]. The study showed that over several decades (from the 1980s and late 1990s), young people started their adult lives two years later on the average than in past decades (1960s and late 1970s). Childbearing also occurred at a later time, increasing the first birth age by oneand-a-half years [4].

Women's sexual health is a determinant of fertility. According to Healthy People 2020, reproductive and sexual health impacts overall health and quality of life and access to these services can decrease the rates of infertility [5]. In many cases, reproductive and sexual health services are the first point of care into the health care system [5]. The services "improve health and reduce costs by covering pregnancy prevention, HIV and sexually transmitted disease (STD) testing and treatment, prenatal care...screening for intimate partner violence and reproductive cancers, provide substance abuse treatment referrals, and counseling on nutrition and physical activity" [5]. Access to quality preventive care helps to reduce rates of infectious diseases, infertility and increases the ability to attain education pursuits [5]. Family planning services allow individuals to plan and space births, prevent unintended pregnancy and improve health outcomes. Publicly funded family planning services in the United States help to prevent 1.94 million unintended pregnancies, with 400,000 of those being teenage girls between the ages of 15-19 [6].

Infertility is the inability to get pregnant after at least a year or longer of unprotected sex regardless of relationship status. Not being able to have a child can reflect the moral standing and in cases, the blame on the woman, who may suffer abuse and ostracism [7]. Infertility carries a social stigma in many countries, with Africa being one of them. As a result, traditional medications and rituals used as intervention methods to conceive and carry children to term [7]. The Centers for Disease Control (CDC) estimates that the number of women aged 15-44 with impaired ability to get pregnant or carry a baby to term is 7.5 million at $12.3 \%$ [8]. About 1 million married women aged 15-44 are infertile at 6.1\%. [8]. Reproductive tract infections, specifically STDs, cause infertility among women. The CDC estimates that undiagnosed and untreated STDs cause at least 24,000 women in the United States each year to become infertile. 
The recommendation is for women to receive annual chlamydia and gonorrhea screening for all sexually active women under the age of 25 , and older women who have a new partner, multiple partners, or a sex partner with an STD.

In the 21st Century, women globally are delaying having children as priorities have changed. Women are prioritizing education and careers before deciding to have a family. Having access to quality preventive services through family planning and screening programs assist in improving health outcomes for women and children. Sexual health can influence fertility at all stages of reproductive health, so knowledge of preventive methods is imperative to reduce the possibility of infertility once the decision is made to plan for a family.

\section{References}

1. Mathews TJ, Hamilton BE (2016) Mean age of mothers is on the rise: United States, 2000-2014. National Center for Health Statistics, USA.
2. Sandberg S, Scovell N (2013) Lean in: Women, work, and the will to lead.

3. Kirk D (1996) Demographic Transition Theory. Population Studies 50(3): 361-387.

4. Bhrolcháin MN, Beaujouan E (2012) Fertility postponement is largely due to rising educational enrolment. Population Studies 66(3): 311327.

5. Office of Disease Prevention and Health Promotion (2017) Reproductive and Sexual Health. In Healthy People 2020.

6. Guttmacher Institute (2011) In Brief: Facts on Publicly Funded Contraceptive Services in the United States. New York, USA.

7. Ember CR, Ember M (2004) Encyclopedia of medical anthropology: Health and Illness in the world's cultures. Kluwer Academic/Plenum Publishers, New York, USA.

8. Centers for Disease Control and Prevention (2016) STDs \& Infertility.

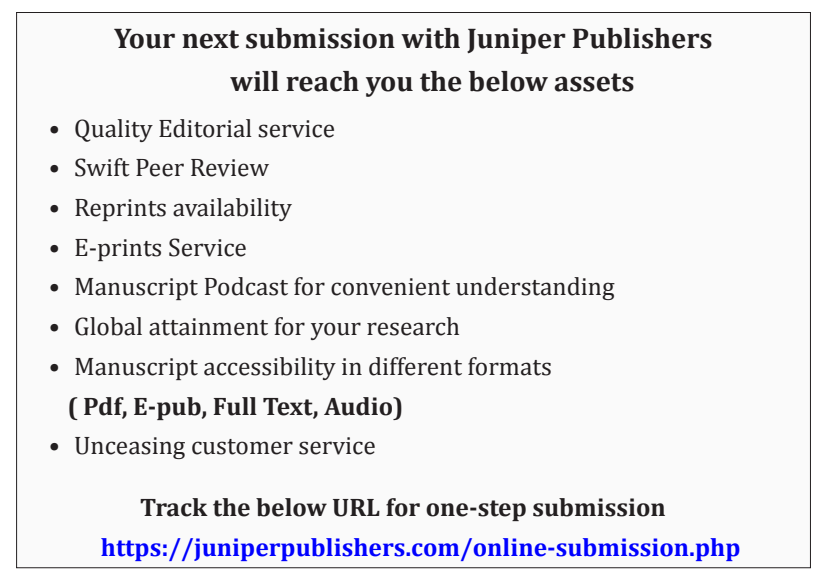

